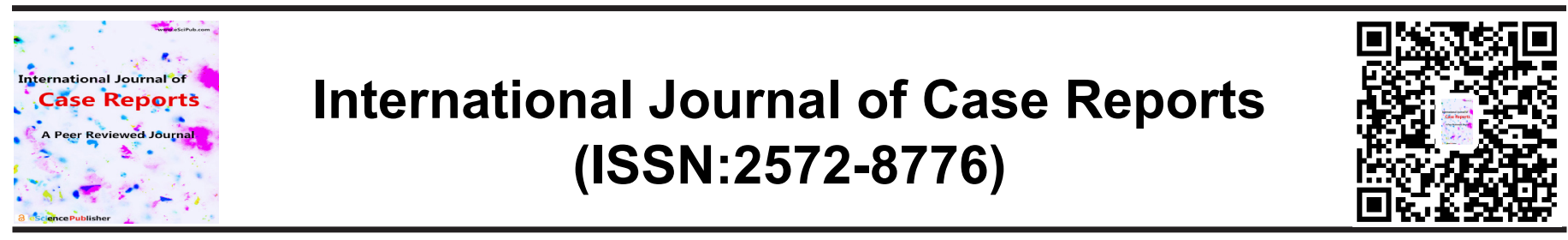

\title{
A NEW TYPE-2-LIKE CALR MUTATION IN ESSENTIAL THROMBOCYTHEMIA
}

Mattia Brescini, dr, Maria Giovanna Loglisci, biotechnologist, Gioia Colafigli, dr, Luisa Bizzoni, dr, Sonia Buffolino, biologist, Daniela Diverio, biologist, Massimo Breccia, dr, Maurizio Martelli, full professor, Stefania Trasarti, dr*

Hematology, Department of Traslational and Precision Medicine, Policlinico Umberto 1, Sapienza University, Rome, Italy.

\section{ABSTRACT}

CALR mutations, together with JAK-2 and MPL ones, are recognized as "driver" mutations in Philadelphia-negative *Correspondence to Author: chronic myeloproliferative neoplasms (MPNs). Most frequent CALR mutations are Type-1 deletions (45-55\% of cases) Stefania Trasarti, dr and type- 2 insertion (32-42\% of cases). These mutations are Hematology, Department of Traslatusually associated with younger age, higher platelet counts, lower leukocyte counts, lower hemoglobin levels and a higher incidence of transformation from ET to MF. Recognizing and describing cases with different mutations can be useful to create ional and Precision Medicine, Policlinico Umberto 1, Sapienza University, Rome, Italy. a database that might help clinicians to include these patients in risk categories and to guide the appropriate therapeutic choices. We report a case of a 77-years old woman who presented a new type-2 like CALR mutation.

KEYWORDS: CALR; Essential Thrombocythemia; Myeloproliferative Neoplasm

\section{How to cite this article:}

Mattia Brescini, Maria Giovanna Loglisci, Gioia Colafigli, Luisa Bizzoni, Sonia Buffolino, Daniela Diverio, Massimo Breccia, Maurizio Martelli, Stefania Trasarti. A NEW TYPE-2-LIKE CALR MUTATION IN ESSENTIAL THROMBOCYTHEMIA. International Journal of Case Reports, 2021; 5:204.

\section{eScîPub}

eSciPub LLC, Houston, TX USA. Website: http://escipub.com/ 


\section{INTRODUCTION}

Philadelphia- negative myeloproliferative neoplasms (Ph-MPNs) are a group of hematopoietic clonal disorders that include polycythemia vera $(P V)$, essential thrombocythemia $(E T)$ and primary myelofibrosis (pMF) ${ }^{[1]}$. Mutations of JAK2 $\mathrm{V} 617 \mathrm{~F}$, together with somatic mutations of the calreticulin gene (CALR) and thrombopoietin receptor gene (MPL) were recognized as "driver" mutations in MPNs. In patients affected by Essential Thrombocythemia, JAK-2 mutations are found in about $60 \%$ of cases, followed by CALR (20-25\%) and MPL (2-3\%) ${ }^{[2]}$.
The two most common CALR mutations are a 52 -bp deletion (Type- $1 ; 45-55 \%$ of the cases) or a 5-bp insertion (Type-2; 32-42\% of the cases). The remaining $15 \%$ of cases include other types of deletions, insertions or both, which are unique or revealed in a small percentage of patients. ${ }^{[3-}$ 4] CALR mutations were usually associated with younger age, higher platelet counts, lower leukocyte counts, lower hemoglobin levels and a higher incidence of transformation from ET to MF when compared to other mutations detected in ET. ${ }^{[5]}$

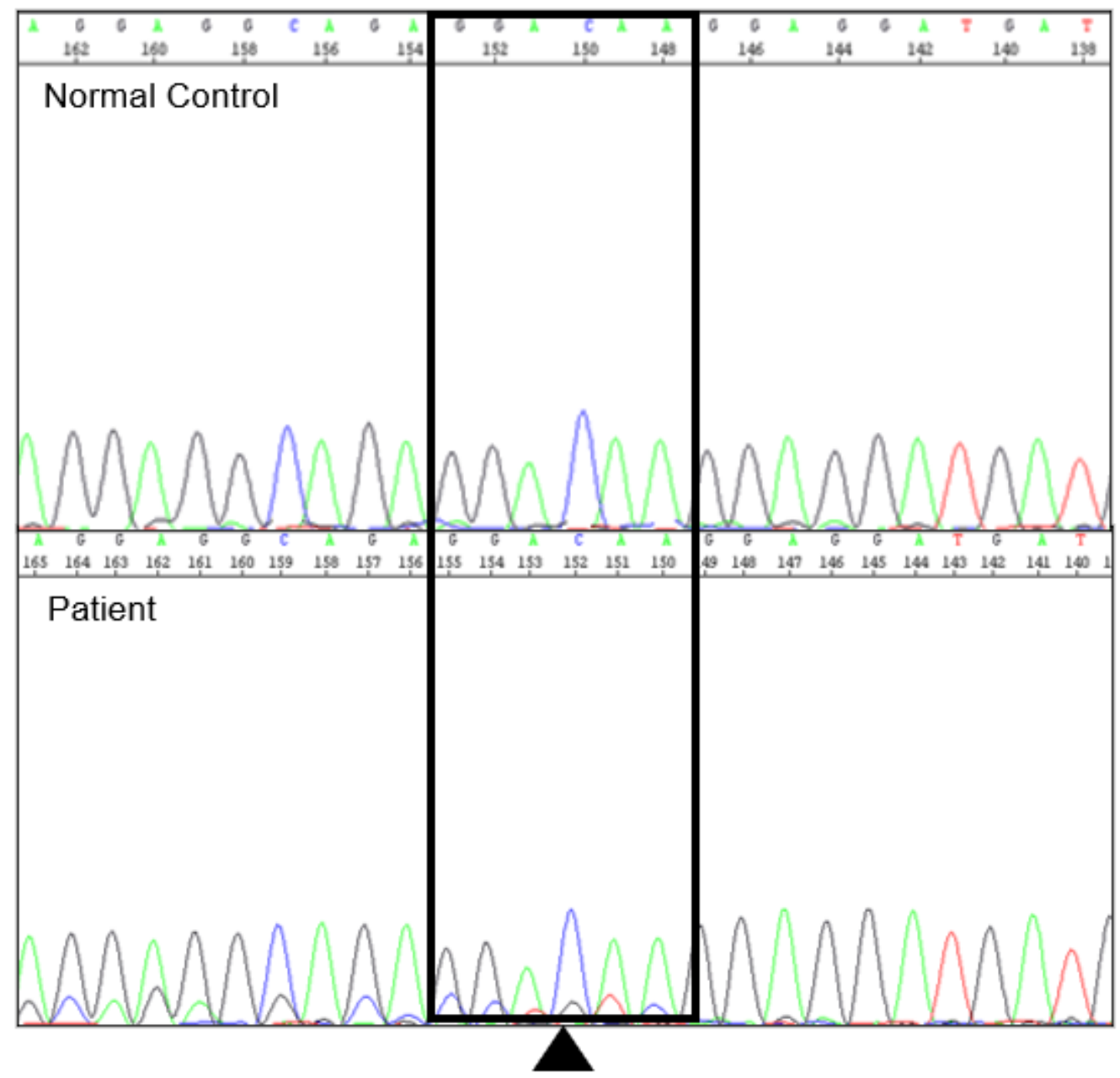

c.1154 delA/insCCTGTC

Wild type sequence

AAEKQMKDKQDEEQRLKEEEEDKKRKEEEEAEDKEDDEDKDEDEEDEEDKEEDEEEDVPGQAKDEL

Mutant sequence

AAEKQMKDKQDEEQRLKEEEEDKKRKEEEEAEDTCRRMMRTKMRMRRMRRTRRKMRRKMSPARPRTSCREACLQ GWTEA

Fig.1 A) Sanger sequencing electrophoregram shows the presence of a new deletion/insertion of $6 \mathrm{bp}$ in the exon 9 of CALR gene. Arrow indicates the position of the deletion/insertion. B) The resulting CALR protein sequence of a wild type and the patient sample. 
In a recent article published in January 2019, the authors reported the development of a rapid lowbudget PCR-based assay in order to screen a large number of MPN patients in the Greek population for frameshift CALR mutations. Thanks to this new assay, the authors were able to find six new rare CALR mutations ${ }^{[6]}$. We want to report here the finding of a new rare mutation, which is not included in the most recent database of Catalogue Of Somatic Mutation In Cancer (COSMIC v92, released August 27 ${ }^{\text {th }}, 2020$ ).

\section{CASE REPORT}

In September 2017, a 77-year old woman referred to our Center with thrombocytosis (995 $\mathrm{x}$ $10 \%$ L) and normocytic anemia (10.1 gr/dl). Biochemical analyses showed a combined iron and cyanocobalamin deficiency that required at first a specific therapy with partial improvement of anemia (in November 2017 hemoglobin increased to $11.3 \mathrm{gr} / \mathrm{dl}$ ) but with persistence of iron deficiency and thrombocytosis. EGDS showed a chronic superficial HP+ gastritis. The patient had no hepatosplenomegaly, JAK-2 V617F mutation was not detected, and the bone marrow biopsy was compatible with ET. Due to the JAK2 negativity, we continued to test the molecular status by Sanger sequencing in an attempt to identify CALR mutations. Sanger showed a new mutation - c.1154_delA/insCCTGTC, K385fs*47 (type 2-like) - which has never been previously described; cytoreductive treatment with hydroxyurea was started immediately in order to reduce thrombocytosis, obtaining a good response (platelet count decreased to $510 \times 10^{9} / \mathrm{L}$ in November 2017). At present, the patient is in good clinical conditions, she is still assuming hydroxyurea with a good control of the platelet count $\left(451 \times 10^{9} / \mathrm{L}\right)$ and resolution of anemia (12.6 gr/dl).

\section{MATERIALS AND METHODS}

To assess the presence of CALR mutations, we studied Genomic DNA obtained from peripheralwhole-blood sample. Genomic regions of interest were amplified by polymerase chain reaction (PCR), using primers spanning exon $9{ }^{[5]}$. PCR products were purified with the use of ExoSAP-
IT TM PCR Product Cleanup Reagent and a sequencing reaction was set up with the use of the BigDye Terminator, 3.1 Cycle Sequencing Kit (Life Technologies). Sequencing products were analyzed using a 3130xl Genetic Analyzer (Applied Biosystems).

\section{DISCUSSION}

In our Center, in the last three years, we screened a total of 154 patients with thrombocytosis; we found CALR mutations in 65 patients (47\%); among them 25 (38\%) were type-1, 37 (57\%) were type-2 and $3(5 \%)$ were atypical mutations (2 type-1-like deletions and 1 type-2-like deletion/insertion that we have described in this paper).

CALR exon 9 somatic mutations are found exclusively in patients with myeloid neoplasms associated with thrombocytosis [3]. They are characterized by a gene signature associated to JAK2 activated pathway suggesting that CALR mutants act through the thrombopoietin receptor [7]. CALR-mutated ET patients, compared with JAK2-mutated ET, usually present younger age, lower hemoglobin levels and white blood cell count with higher platelet count ${ }^{[8]}$. Prognostic implications of CALR type 1- and type 2-like mutations have been investigated, showing that type 2-like mutations are associated with reduced thrombotic events and a decreased risk of myelofibrotic transformation compared to type 1like and JAK2 mutated patients ${ }^{[5]}$. Different clinical behaviour could be related to the variant mutation but large cohort of patients with different type 1-like or 2-like should be analysed in detail to define the long-term prognostic implications.

Recognizing and describing ET cases with different somatic mutations of CALR and the other driver genes and updating the COSMIC database with these new findings, might help clinicians in the next future to include these patients in different risk categories and to guide the most appropriate therapeutic choices.

Acknowledgement: This section includes a brief acknowledgment of people, grant details, funds

\section{REFERENCES}

IJCR: https://escipub.com/international-journal-of-case-reports/ 
[1] Arber DA, Orazi A, Hasserjian R, et al. The 2016 revision to the World Health Organization classification of myeloid neoplasms and acute leukemia. Blood 2016; 127(20): 2391-2405

[2] Baxter EJ, Scott LM, Campbell PJ, East C, Fourouclas N, Swanton S, Vassiliou GS, Bench AJ, Boyd EM, Curtin N, Scott MA, Erber WN, Green AR; Cancer Genome Project. Acquired mutation of the tyrosine kinase JAK2 in human myeloproliferative disorders. Lancet 2005; 365 (9464): 1054-1061

[3] Klampfl T, Gisslinger H, Harutyunyan AS, Nivarthi $\mathrm{H}$, Rumi E, Milosevic JD, Them NC, Berg T, Gisslinger B, Pietra D, Chen D, Vladimer GI, Bagienski K, Milanesi C, Casetti IC, Sant'Antonio E, Ferretti V, Elena C, Schischlik F, Cleary C, Six M, Schalling M, Schönegger A, Bock C, Malcovati L, Pascutto C, Superti-Furga G, Cazzola M, Kralovics R. Somatic mutations of calreticulin in myeloproliferative neoplasms. New England Journal of Medicine 2013; 369(25): 2379-2390

[4] Rotunno G, Mannarelli C, Guglielmelli P, et al. Impact of calreticulin mutations on clinical and hematological phenotype and outcome in essential thrombocythemia. Blood 2014. 123: 1552-1555.

[5] Pietra D, Rumi E, Ferretti V, Di Buduo CA, Milanesi $C$, Cavalloni $C$, et al. Differential clinical effects of different mutation subtypes in CALR- mutant myeloproliferative neoplasms. Leukemia 2016; 30: 431-438

[6] Giannopoulos A, Rougkala N, Loupis T, Mantzourani M, Viniou NA, Variami E, Vassilakopoulos TP, Dryllis G, Kotsianidis I, Gougopoulou T, Politou M, Konstantopoulos K, Vassilopoulos G. Detection of CALR Mutations Using High Resolution Melting Curve Analysis (HRM-A); Application on a Large Cohort of Greek ET and MF Patients. Mediterranean Journal of Hematology and Infectious Diseases. 2019 Jan 1;11(1): e2019009. doi: 10.4084/MJHID.2019.009. PMID: 30671215; PMCID: PMC6328041.

[7] Rampal R, Al-Shahrour F, Abdel-Wahab O, Patel JP, Brunel JP, Mermel $\mathrm{CH}$ et al. Integrated genomic analysis illustrates the central role of JAKSTAT pathway activation in myeloproliferative neoplasm pathogenesis. Blood 2014; 123: e 123e133

[8] Rumi E, Pietra D, Ferretti V, Klampfl T, Harutyunyan AS, Milosevic JD, et al. JAK2 or CALR mutation status defines subtypes of essential thrombocythemia with substantially different clinical course and outcomes. Blood 2014; 123: 1544-1551

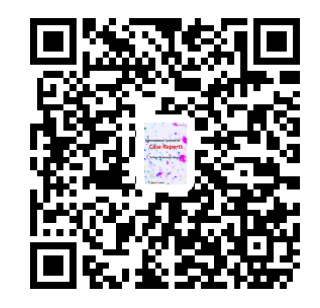

